\title{
Targeted Injection of a Truncated Form of Tissue Inhibitor of Metalloproteinase 3 Alters Post-Myocardial Infarction Remodeling ${ }^{\mathbb{S}}$
}

\author{
David C. Lobb, Heather Doviak, Gregory L. Brower, Eva Romito, Jason W. O’Neill, \\ Stephen Smith, James A. Shuman, Parker D. Freels, Kia N. Zellars, Lisa A. Freeburg, \\ Aarif Y. Khakoo, (1)TaeWeon Lee, and Francis G. Spinale \\ Cardiovascular Translational Research Center, University of South Carolina School of Medicine and the WJB Dorn Veteran \\ Affairs Medical Center, Columbia, South Carolina (D.C.L., H.D., G.L.B., E.R., J.A.S., P.D.F., K.N.Z., L.A.F., F.G.S.) and Amgen, \\ Metabolic Disorders, South San Francisco, California (J.W.O., S.S., A.Y.K., T.L.)
}

Received April 13, 2020; accepted August 18, 2020

\begin{abstract}
Infarct expansion can occur after myocardial infarction (MI), which leads to adverse left ventricular (LV) remodeling and failure. An imbalance between matrix metalloproteinase (MMP) induction and tissue inhibitors of MMPs (TIMPs) can accelerate this process. Past studies have shown different biologic effects of TIMP-3, which may depend upon specific domains within the TIMP-3 molecule. This study tested the hypothesis that differential effects of direct myocardial injections of either a full-length recombinant TIMP-3 (F-TIMP-3) or a truncated form encompassing the $\mathrm{N}$-terminal region (N-TIMP-3) could be identified post-MI. MI was induced in pigs that were randomized for $\mathrm{Ml}$ injections $(30 \mathrm{mg})$ and received targeted injections within the Ml region of F-TIMP-3 $(n=8), \mathrm{N}-\mathrm{TIMP}-3(n=9)$, or saline injection (MI-only, $n=11$ ). At 14 days post-MI, LV ejection fraction fell post-MI but remained higher in both TIMP-3 groups. Tumor necrosis factor and interleukin-10 mRNA increased by over 10 -fold in the MI-only and N-TIMP-3 groups but were reduced with F-TIMP-3 at this post-Ml time point. Direct Ml injection of either a full-length or truncated form of TIMP-3 is sufficient to favorably alter the course of post-Ml remodeling. The functional and differential
\end{abstract}

relevance of TIMP-3 domains has been established in vivo since the TIMP-3 constructs demonstrated different MMP/cytokine expression profiles. These translational studies identify a unique and more specific therapeutic strategy to alter the course of $L V$ remodeling and dysfunction after Ml.

\section{SIGNIFICANCE STATEMENT}

Using different formulations of tissue inhibitor of matrix metalloproteinase-3 (TIMP-3), when injected into the myocardial infarction (MI) region, slowed the progression of indices of left ventricular (LV) failure, suggesting that the $\mathrm{N}$ terminus of TIMP-3 is sufficient to attenuate early adverse functional events post-Ml. Injections of full-length recombinant TIMP-3, but not of the $\mathrm{N}$-terminal region of TIMP-3, reduced relative indices of inflammation at the mRNA level, suggesting that the C-terminal region affects other biological pathways. These unique proof-ofconcept studies demonstrate the feasibility of using recombinant small molecules to selectively interrupt adverse LV remodeling post-MI.

\section{Introduction}

A structural milestone in the progression of heart failure after a myocardial infarction (MI) is left ventricular (LV)

This work was supported by National Institutes of Health National Heart, Lung, and Blood Institute [Grants HL111090 and HL113352], a Merit Award from the Veterans' Affairs Health Administration, and a basic research grant from Amgen, Inc.

J.W.O., S.S., A.Y.K., and T.L. are employees of Amgen Inc. that manufacture drugs for a wide range of diseases, including cardiovascular disease. The other authors declare that they have no competing interests. The recombinant TIMP-3 formulations were furnished as an material transfer agreement from Amgen to F.G.S.

https://doi.org/10.1124/jpet.120.000047.

S This article has supplemental material available at jpet.aspetjournals.org. remodeling, which can be defined as changes in LV geometry and structure (Sutton and Sharpe, 2000; Weir et al., 2006; Spinale, 2007; Konstam et al., 2011). Specifically, this adverse remodeling process is characterized by continuous turnover of the extracellular matrix (ECM) within the MI region, causing mural wall thinning, LV chamber dilation, and eventually pump dysfunction (Mukherjee et al., 2003; Spinale, 2007; Lindsey and Zamilpa, 2012). One biologic system that is active in the post-MI context is a family of ECM proteases, the matrix metalloproteinases (MMPs) (Mukherjee et al., 2003; Spinale, 2007; Lindsey and Zamilpa, 2012), whereby induction and release of MMPs has been demonstrated in patients post-MI and associated with adverse LV remodeling (Webb et al., 2006). Basic studies utilizing transgenic

ABBREVIATIONS: BAX, Bcl-2-associated protein; BCL2, B-cell lymphoma 2; Ct, cycle times; ECM, extracellular matrix; F-TIMP-3, full-length recombinant TIMP-3; IL, interleukin; LSD, least-significant difference; LV, left ventricular; MCP1, monocyte chemoattractant protein-1; MI, myocardial infarction; MIP1A, macrophage inflammatory protein-1 alpha; MMP, matrix metalloproteinase; N-TIMP-3, N-terminal region TIMP-3; PCR, polymerase chain reaction; PSR, picrosirius red; SMA, smooth muscle actin; TIMP, tissue inhibitor of metalloproteinase; TNF, tumor necrosis factor. 
constructs or pharmacological strategies provided mechanistic evidence that modulating MMP activity would favorably alter the course of post-MI remodeling (Heymans et al., 1999; Lindsey et al., 2002; Mukherjee et al., 2003; Spinale, 2007; Kandalam et al., 2010; Lindsey and Zamilpa, 2012). However, clinical translation of these studies have encountered problematic issues, which include the ability of systemic delivery of pharmacological MMP inhibitors reaching therapeutic levels and concerns regarding off-target effects (Peterson, 2004; Hudson et al., 2006; Dormán et al., 2010). An alternative therapeutic approach would be to target MMP activity within the MI region specifically and thus avoid potential systemic effects. Accordingly, this project used a relevant large animal post-MI model (Dixon and Spinale, 2009) and a localized targeted approach to interfere with MMP proteolytic activity. Endogenous MMP inhibition is achieved through the synthesis and release of the tissue inhibitors of MMPs (TIMPs) (Douglas et al., 1997; Bourboulia and Stetler-Stevenson, 2010; Brew and Nagase, 2010). There are four known TIMPs, and past studies have identified that a relative MMP/TIMP imbalance occurs within the MI region, thus favoring ECM proteolysis and instability and post-MI remodeling (Peterson et al., 2000; Mukherjee et al., 2003; Wilson et al., 2003; Spinale, 2007; Lindsey and Zamilpa, 2012). However, the effects of individual TIMPs are not uniform in terms of MMP inhibitory profiles, processing of biologic signaling cascades, and effects upon growth/proliferation/viability (Abbate et al., 2002; Lovelock et al., 2005; Melendez-Zajgla et al., 2008; Hammoud et al., 2009; Troeberg et al., 2009; Lu et al., 2011). For example, unique features of TIMP-3 include a high affinity to bind to the ECM (Leco et al., 1994; Yu et al., 2000), an influence on cytokine processing (Troeberg et al., 2009; Bourboulia and Stetler-Stevenson, 2010; Brew and Nagase, 2010), and altering fibroblast phenotype in vitro (Yang and Hawkes, 1992; Lovelock et al., 2005; Shinde and Frangogiannis, 2014). Moreover, transgenic deletion of TIMP-3 causes adverse remodeling, acceleration to heart failure, and reduced survival (Kandalam et al., 2010; Hammoud et al., 2011). These previous studies provide a strong rationale for pursuing the concept that augmentation of TIMP-3 would be a potential therapeutic strategy post-MI. Specifically, this project was developed around the central hypothesis that a localized, targeted augmentation of TIMP-3 within the MI region would favorably alter adverse LV remodeling. Thus, the first objective of this study was to directly inject a full-length recombinant TIMP-3 into the MI region and quantify the effects upon the natural history of post-MI remodeling.

The structural domains of TIMPs can be divided into the N-terminal and C-terminal subdomains, whereby the global MMP inhibitory component appears to reside in the $\mathrm{N}$-terminal domain (Douglas et al., 1997; Brew and Nagase, 2010). With respect to TIMP-3, past in vitro studies have demonstrated that mutations within the N-terminal domain, while altering MMP inhibitory effects, did not alter proteolytic mediated cytokine processing (Wei et al., 2005). These studies led us to postulate that the $\mathrm{N}$-terminal domain of TIMP-3 may be sufficient to blunt MMP activity and hence modify post-MI remodeling. Accordingly, the second objective of this study was to perform targeted injections of a truncated form of TIMP-3 containing the $\mathrm{N}$-terminal domain and perform comparative studies of post-MI remodeling.

\section{Methods}

In this study, both a full-length TIMP-3 recombinant protein, referred to as F-TIMP-3, and a truncated form of TIMP-3 containing the N-teminal domain of TIMP-3, referred to as N-TIMP-3, were used. The TIMP-3 structure and sequences for these constructs are shown in Figure 1A, and the methodology for the construction of these TIMP-3 variants is provided in Supplemental Methods. Initial in vitro and in vivo studies were performed to validate the TIMP-3 formulations in terms of MMP inhibition and myocardial localization as provided in detail in the Supplemental Methods. Briefly, using an MMP fluorescent peptide assay (Spinale et al., 2008), inhibition in MMP activity occurred with increasing concentrations of either F-TIMP-3 or N-TIMP-3 (Fig. 1B) with an approximate IC $_{50}$ of $2-6 \mu \mathrm{g} / \mathrm{ml}(0.4-5$ $\mathrm{nM}$ ), indicating that both proteins inhibited MMP activity in the manner consistent with native TIMP-3 (Douglas et al., 1997; (Moore et al., 2012)). Studies were then carried out to develop a dose and localization of the F-TIMP-3 or N-TIMP-3 injections. In the subsequent efficacy studies, equivalent concentrations of the TIMP-3 formulations were injected into the MI region to examine the effects on LV geometry and function. For the animal model, adult pigs were used, as MI induction in this species results in uniformity of MI size and temporal changes in LV geometry (Mukherjee et al., 2003, 2008; Dixon and Spinale, 2009; Eckhouse et al., 2014). All animals were treated and cared for in accordance with the National Institutes of Health Guide for the Care and Use of Laboratory Animals (eighth edition, Washington, DC, 2011), and all protocols were approved by the University of South Carolina's Institutional Animal Care and Use Committee. Serial studies were carried out until 14 days post-MI, as this time period encompasses a rapid change in LV geometry and function in both animals and patients (Mukherjee et al., 2003; Webb et al., 2006). After the final set of LV function measurements, sections of the MI region were subjected to mRNA analysis for MMP/TIMP and cytokine levels as well as histochemistry.

Development of TIMP-3 Myocardial Injection Strategy and Validation. Targeted myocardial injections of the TIMP-3 formulations were performed within the LV free wall of mature pigs $(25 \mathrm{~kg}$, Yorkshire; Hambone Farms, Orangeburg, SC). The pigs $(n=10)$ were anesthetized with isoflurane ( $2 \%)$, and through a left thoracotomy, the $\mathrm{LV}$ free wall was exposed. A six-point injection grid $(2 \times 2 \mathrm{~cm}$, uniform point distances of $0.5 \mathrm{~cm}$ ) was temporarily sutured to the epicardial surface below the origin of the first two obtuse marginal arteries of the circumflex artery (schematic shown in Fig. 1C). This targeted injection region is contained within the myocardial region for coronary ligation and subsequent MI induction. The F-TIMP-3 or N-TIMP-3 formulations were diluted in sterile saline to provide final concentrations of 2 , 5 , or $10 \mathrm{mg} / 100 \mu \mathrm{l}$ (injection site volume $100 \mu \mathrm{l}$, six injection sites; total injection volume $600 \mu \mathrm{l}$; BD Ultra-Fine $31 \mathrm{G}$ ). The specific approaches and results from these studies are provided in Supplemental Methods. Using these results, and to ensure dosing equivalency with respect to mass and volume, a $5 \mathrm{mg} /$ injection dose was selected for subsequent in vivo studies. Using the six-point injection pattern yielded a total myocardial delivery of $30 \mathrm{mg}$ of either F- or N-TIMP-3. Although absolute myocardial concentration computations require several assumptions, the total injected myocardial region was $4 \mathrm{ml}(2 \times 2 \times$ $1 \mathrm{~cm}$, specific gravity of $1 \mathrm{~cm}^{3} / \mathrm{ml}$ ), resulting in an initial delivery of $750 \mu \mathrm{g} / \mathrm{ml}$, thus reflective of a $100 \times$ concentration from the computed $\mathrm{EC}_{50}$ for the TIMP formulations.

The next set of studies more carefully examined the spatial distribution and temporal retention of the TIMP-3 formulations in the targeted myocardial injection region. Specifically, F-TIMP-3 or N-TIMP-3 were fluorescently labeled as detailed in the Supplemental Methods. Pigs (25 kg, $n=12$ ) underwent targeted myocardial injections of fluorescently labeled F-TIMP-3 or N-TIMP-3 (six-injection pattern) as described in the previous section, whereby the LV was harvested immediately postinjection or at 3 and 5 days postinjection, and circumferential LV sections were subjected to epiillumination imaging as detailed in the Supplemental Methods. 


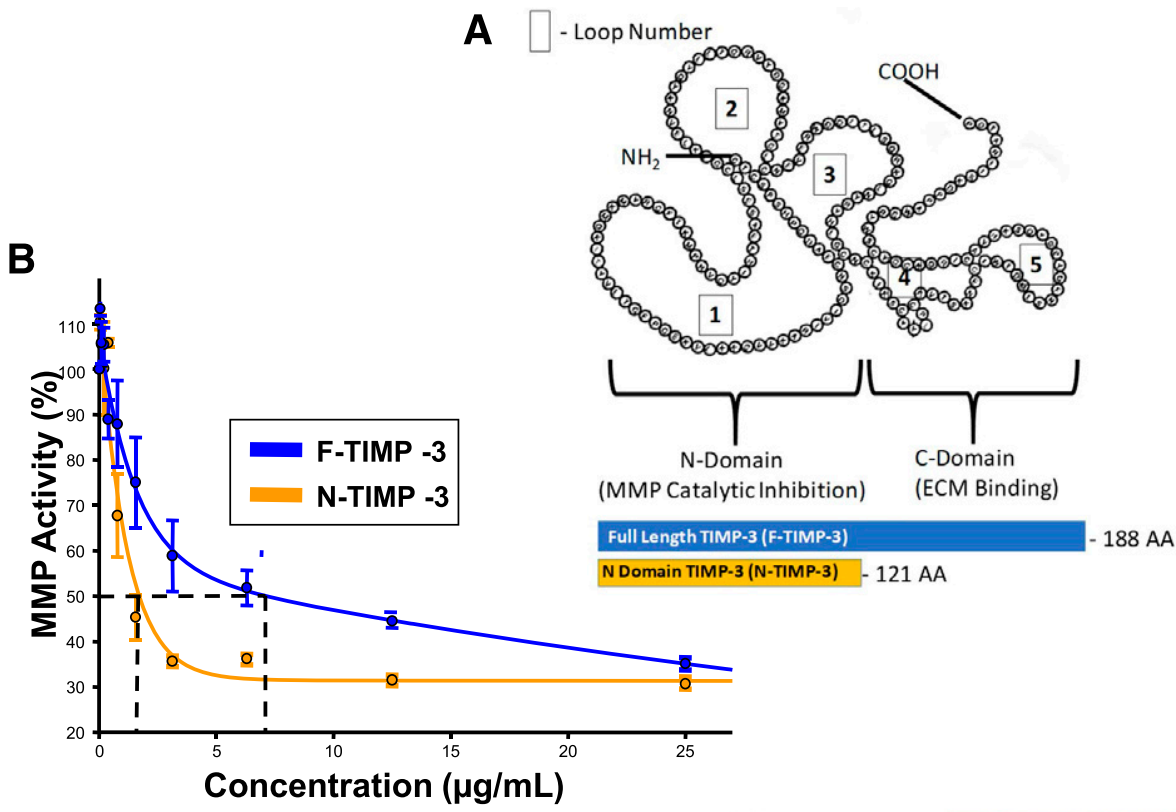

D

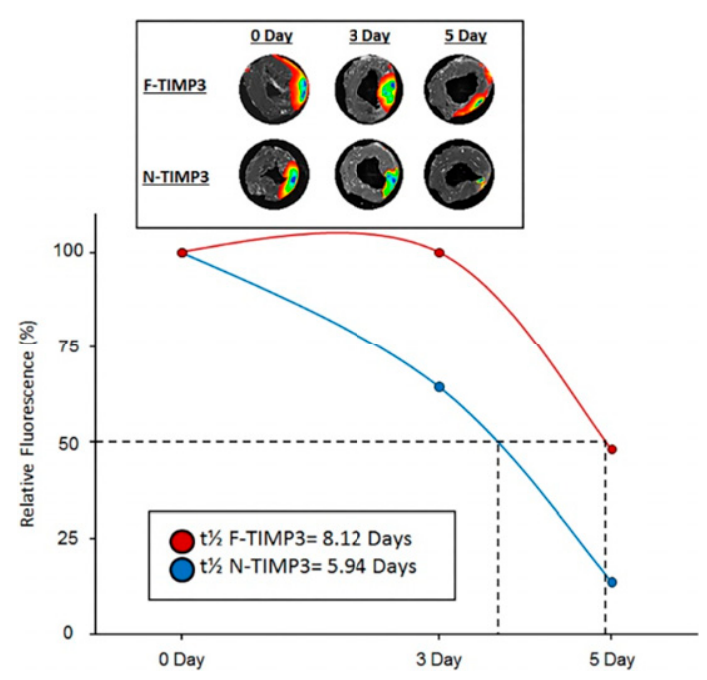

Fig. 1. (A) Structure/sequence for F-TIMP-3. The domain that comprises binding and inhibition of active MMPs is the $\mathrm{N}$ terminus, and a truncated peptide containing this entire region was synthesized (N-TIMP-3). (B) Inhibitory profiles for both F-TIMP-3 and N-TIMP-3 were examined using an MMP fluorescent activity assay. The dashed lines reflect the respective $\mathrm{IC}_{50}$. (C) Schematic of six-point injection site for the TIMP-3 formulations. OM1 and OM2, obtuse marginal coronary arteries 1 and 2. (D) LV myocardial injections of fluorescently labeled F-TIMP-3 or N-TIMP-3 (5 $\mathrm{mg}$ ) demonstrated localization in the targeted area (3 and 5 days postinjection) with computed retention time for each TIMP3 shown.
A representative set of $\mathrm{LV}$ images from each region with injection of each TIMP-3 formulation immediately after injection and at 3 and 5 days postinjection is shown in Figure 1D. Using an exponential fit model, the computed F-TIMP-3 retention half-life was approximately 8 days, and for N-TIMP-3, it was approximately 6 days.

MI Induction and TIMP-3 Injections: Randomization and Uniformity in MI Size. For this protocol, pigs ( $n=26,20 \mathrm{~kg}$, male) were randomized to one of three different groups: MI and saline injections (MI/saline; $100 \mu \mathrm{l}$ injection/six injection sites, $n=9$ ), MI and F-TIMP-3 injections (MI/F-TIMP-3; $30 \mathrm{mg}$ total injection, $n=8$ ), and MI and N-TIMP-3 injections (MI/N-TIMP-3; $30 \mathrm{mg}$ total injection, $n=9$ ). The pigs were randomized prior to surgery using a random number table, and the treatment code was not broken until the completion of the entire protocol and analysis. The pigs were anesthetized as described in the previous section, and after a thoracotomy, a vascular access catheter ( $6 \mathrm{Fr}$; Access Technologies) was placed in the thoracic aorta and attached to a subcutaneous port for blood sample collection and measurement of troponin I (ELISA, Cat \#KT-641; Kamiya Biomedical Company, Seattle, WA). Plasma troponin I measurements were obtained prior to MI induction (baseline), at 24 hours and 72 hours post-MI, and at 14 days post-MI. MI induction was achieved by ligating the obtuse marginal branches 1 and 2 at the origin just below the circumflex artery as described previously (Mukherjee et al., 2008). A cohort $(n=5)$ of age/weight matched pigs were treated in identical fashion (sham procedures) and served as referent controls for myocardial biochemistry and histology.

Serial Measurements of LV Geometry and Function, Referent Controls, and Sampling. An outline of the experimental design is shown in Figure 2A with the sampling points identified. The day before randomization and MI induction, the animals were sedated (diazepam, $200 \mathrm{mg}$ by mouth; Barr Laboratories, Pomona, NY), and echocardiography was performed (GE VIVID 7 Dimension Ultrasound System: M4S 1.5-4.3 MHz active matrix array sector transducer probe) to measure LV volumes, left atrial area, posterior LV free wall thickness, and ejection fraction as described previously (Mukherjee et al., 2003, 2008; Eckhouse et al., 2014). In addition, mitral valve inflow velocities and tissue Doppler were used to compute an estimate of pulmonary capillary wedge pressure (Zile et al., 2011). The pigs were returned to the laboratory under identical sedation/study conditions at $1,3,7$, and 14 days post-MI. At the completion of the study interval, the pigs were again anesthetized, and the LV region containing the MI region was harvested. 


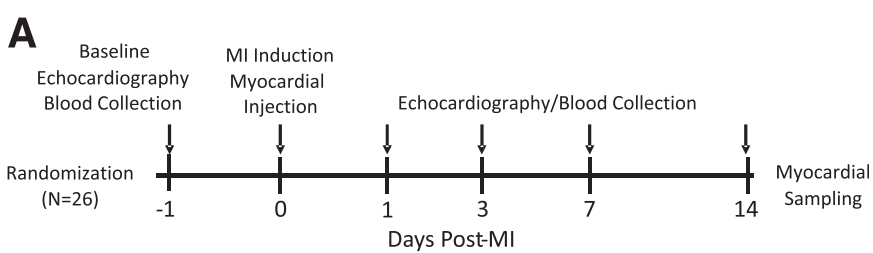

B

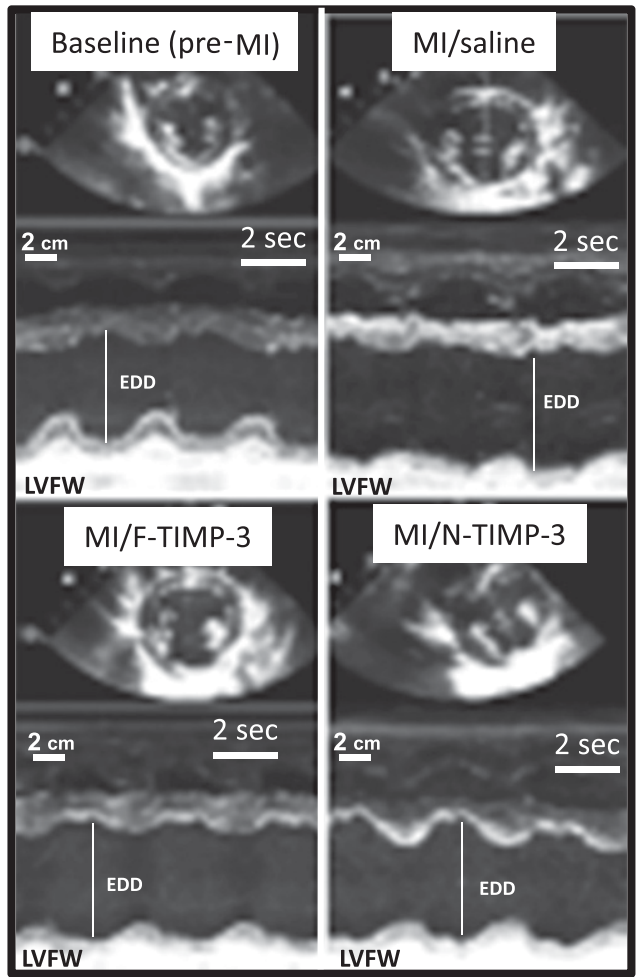

Fig. 2. (A) Experimental design for LV function and myocardial injection/ sampling. (B) Representative LV short axis views (top) and twodimensional targeted M-Mode images (bottom) at baseline (pre-MI) and at 14 days post-MI for the three groups. LV volumetric measurements were computed using LV long axis as well as short axis images. These echocardiograms exemplify the degree of $\mathrm{LV}$ dilation and loss of $\mathrm{LV}$ posterior free wall (LVFW) motion as well as wall thinning at 14 days postMI. In both TIMP-3 injection groups, the magnitude of LV dilation and LVFW wall thinning were attenuated. Summary results for LV geometry and function are in Figure 3. EDD, LV end-diastolic dimension.

Myocardial MMP/TIMP/Cytokine mRNA, Histochemistry, and MMP Activity. RNA was extracted from the LV samples (Experion Automated Electrophoresis System; Bio-Rad Laboratories, Hercules, CA), reverse transcribed (iScript cDNA Synthesis Kit; BioRad), and cDNA amplified with gene/pig specific primer/probe sets $\left(\mathrm{RT}^{2}\right.$ Profiler PCR Custom Array; Qiagen), which are presented in Supplemental Table 1 . The array was designed to contain primers for representative MMP types, all four TIMPs, the fibrillar collagens (collagen I and III), and inflammatory cytokines identified to be relevant to post-MI remodeling (Dewald et al., 2005; Frangogiannis, 2012). In addition, indices of the Bcl-2 family, which regulate mitochondrial apoptotic factors Bcl-2-associated protein (BAX), and B-cell lymphoma 2 (BCL2), were also included in this analysis (Abbate et al., 2002). The reaction was performed ( $\mathrm{RT}^{2} \mathrm{SYBR}$ Green@qPCR Mastermix; Qiagen) and quantified by real-time polymerase chain reaction (PCR) (CFX96 real-time PCR detection system; Bio-Rad). In addition to the targeted PCR measurements, a large porcine cytokine/ receptor PCR array was used (330231 PASS-011ZD; Qiagen), which contained 84 inflammatory cytokines and cytokine receptors (Supplemental Table 2). The real-time PCR fluorescence signal was converted to cycle times (Ct) normalized to glyceraldehyde-3-phosphate dehydrogenase $(\Delta \mathrm{Ct})$. All PCR assays were performed in duplicate.

LV samples were formalin fixed, embedded, sectioned $(7 \mu \mathrm{m})$, and stained with H\&E and picrosirius red (PSR) for fibrillar collagen, whereby the percent area of collagen was computed using computer assisted morphometry (Nikon E600 with Q imaging software and Image-Pro Plus Version 4.5). For both the MI and remote LV sections, an average of 15 random fields were digitized from three independent sections and computed as a composite value for each pig. Additional LV sections were stained with hematoxylin and eosin, and histopathological examination of the targeted MI region was performed. Additional LV sections were used for immunostaining to localize cells that stained positive for $\alpha$-smooth muscle actin (SMA, 1:100; A5228; Sigma) as well as for macrophages using a previously validated antisera in porcine formalin fixed tissue (ionized calcium-binding adaptor protein-1 (IBA-1), 1:2000, 019-19741; Wako Chemicals) (Bolz et al., 2016). Using quantitative morphometry, the percent area staining for SMA and IBA-1 was computed for both the MI and remote regions.

LV samples from the MI region were homogenized and subjected to MMP activity assays using the global MMP substrate described in the previous section. Briefly, LV samples were homogenized using ice cold buffer [cacodylic acid (10 mM/l), $\mathrm{NaCl}(0.15 \mathrm{M} / \mathrm{l}), \mathrm{ZnCl}(20 \mathrm{mM} / \mathrm{l}), \mathrm{NaN}_{3}$ (1.5 mM/l), and 0.01\% Triton X-100 (pH 5.0)] and centrifuged, and LV extracts $(10 \mu \mathrm{g}$; Pierce BCA Protein Assay Kit, Cat \#23225; Thermo Scientific) were incubated at $37^{\circ} \mathrm{C}$ with the global MMP substrate $\left(37^{\circ} \mathrm{C}\right.$ for 6 hours) and fluorescence recorded. Using the same conditions, LV extracts $(10 \mu \mathrm{g})$ were also incubated with a fluorogenic membrane type 1-matrix metalloproteinase (MT1-MMP) specific substrate (0.06 $\mu \mathrm{M}$, Cat \#444258; Millipore) and fluorescence measured at 6 hours (328/400 nm, FLUOstar; BMG Laboratories).

Computation and Data Analysis. Statistical analyses were performed (STATA Corp, College Station, TX), whereby LV geometry and function were initially examined by a two-way ANOVA in which time and treatment were considered the main effects. Post hoc separation after ANOVA was performed using pairwise comparisons with a least-significant difference (LSD) analysis (LSD module; STATA). If the assumptions regarding equal variances between groups or normality in the data distribution were not met, then a nonparametric pairwise comparison between groups at matched time points was performed using Wilcoxon (nonparametric module; STATA). For example, this was encountered for the cytokine mRNA measurements. Results are presented as means \pm S.E.M., and values of $P<0.05$ were considered to be statistically significant.

\section{Results}

TIMP-3 Injections Attenuate Adverse LV Remodeling Post-MI. Plasma troponin I values were equivalent across the randomized treatment groups (Fig. 3). Specifically, a significant and equivalent surge in plasma troponin I occurred at 24 hours post-MI and returned to near baseline values in all groups at later post-MI time points. Thus, a uniform and consistent magnitude of myocardial injury was induced and thereby removed this potential confounding factor from the experimental design. Key indices of LV function and geometry are shown in Figure 3. Representative LV echocardiograms are shown in Figure 2B, and a summary of key indices of LV function and geometry are shown in Figure 3. LV end-diastolic volume, a reflection of post-MI remodeling, increased in a time-dependent manner post-MI in all groups but was reduced in both the F-TIMP-3 and N-TIMP3 groups at later post-MI time points. LV posterior wall thickness, another index of MI remodeling, decreased in a time-dependent manner post-MI but was attenuated in both 

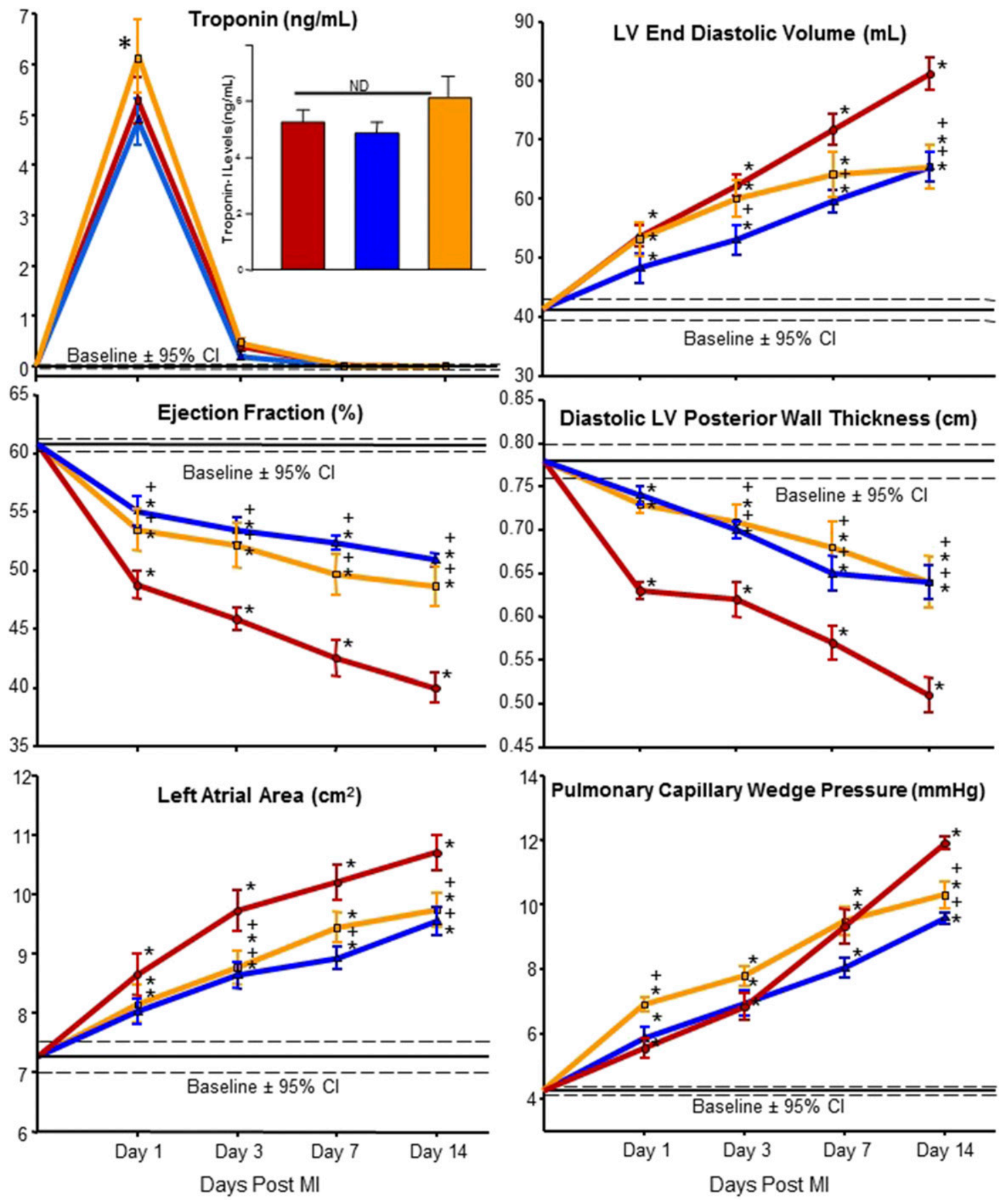

Fig. 3. Plasma troponin-I levels were determined prior to MI induction (baseline) at 24 and 72 hours post-MI induction and at terminal study on post-MI day 14 in pigs randomized to the three treatment groups. Plasma troponin-I levels were near nondetectable levels at baseline, spiked at 24 hours postMI, and had returned to within baseline ranges by 3 and 14 days post-MI in all groups. The inset panel presents the plasma troponin-I levels at 24 hours post-MI for all groups, and, although they increased from baseline, they were equivalent (ND, no significant difference). LV end-diastolic volume increased as a function of time post-MI but was reduced at later time points in both TIMP-3 formulation groups. These changes were paralleled by an improvement in LV ejection fraction and wall thickness (at MI region) in both TIMP-3 formulation groups. Additional indices of post-MI remodeling, as reflected by left atrial geometry (area) and filling pressure (pulmonary capillary wedge pressure), were reduced at later post-MI time points in both TIMP-3 formulation groups. $* P<0.05$ vs. baseline values; ${ }^{+} P<0.05$ vs. MI/saline; determined by post hoc adjusted pairwise comparisons, LSD test. Sample sizes: MI/saline $n=9$, MI/F-TIMP-3 $n=8$, MI/N-TIMP-3 $n=9$. 95\% CI, 95\% confidence interval. 

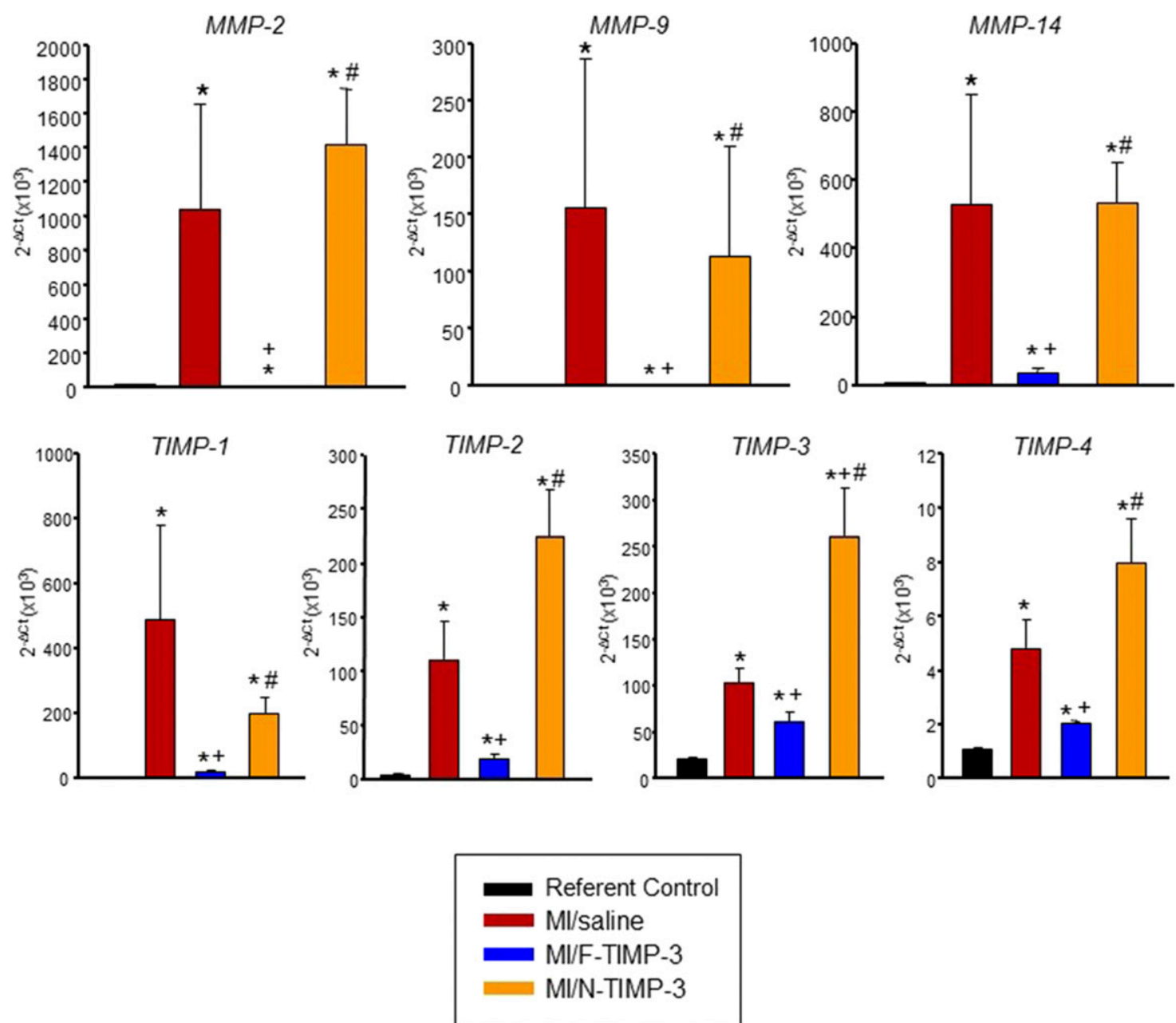

Fig. 4. A pattern of expression emerged whereby mRNA levels for MMPs and TIMPs were increased in the MI/saline and MI/N-TIMP-3 groups and reduced in the MI/F-TIMP-3 group. Of note, relative TIMP-3 mRNA levels were increased to the greatest degree in the MI/N-TIMP-3 group. $* P<0.05$ vs. control values; ${ }^{+} P<0.05$ vs. MI/saline; ${ }^{\#} P<0.05$ vs. MI/F-TIMP-3, determined by nonparametric Wilcoxon test. Sample sizes: MI/saline $n=9$, MI/ F-TIMP-3 $n=8$, MI/N-TIMP-3 $n=9$, referent control $n=5$.

TIMP-3 injection groups. LV ejection fraction, a measure of global pump performance, fell significantly post-MI, and this impairment in LV pump function was improved in both TIMP3 groups. Left atrial area, which reflects changes in LV filling pressure and remodeling, increased post-MI but was reduced at later post-MI time points in the TIMP-3 injection groups. Similarly, pulmonary capillary wedge pressure, which is reflective of left atrial pressures and hence changes in LV filling and remodeling, increased post-MI and was attenuated at late post-MI time points with the TIMP-3 injections. For all of these measures of LV function and geometry, the ANOVA revealed a significant time-treatment interaction $(P<0.05)$, which indicates that TIMP-3 treatment altered the course of post-MI remodeling. Although each of the TIMP-3 formulation injections was different from MI/saline values at key post-MI time points, adjusted pairwise comparisons revealed no differences in these effects between the F-TIMP-3 and N-TIMP-3 formulations.

Differential Effects on MMP/TIMP mRNA Expression with TIMP-3 Injections. Relative mRNA levels for MMP-2, MMP-9, and MMP-14 were robustly increased in the MI/saline group compared with referent control values but were significantly suppressed in the F-TIMP-3 group (Fig. 4). In contrast,
mRNA levels for these MMP types were similar to MI/saline values in the N-TIMP-3 group. Similarly, mRNA levels for MMP-13 were increased from referent control values in the $\mathrm{MI} /$ saline and MI/N-TIMP-3 groups $(0.9 \pm 0.1,3.7 \pm 1.5,3.3 \pm$ $0.72^{-\Delta \mathrm{Ct}} \times 10^{3}, P<0.05$ respectively) and fell to control values in the MI/F-TIMP-3 group $\left(0.9 \pm 0.1^{-\Delta \mathrm{Ct}} \times 10^{3}, P<0.05\right)$. Relative expression levels for endogenous TIMP-1, -2, -3, and -4 were higher than referent control in the MI/saline group and were uniformly reduced in the F-TIMP-3 group (Fig. 4). However, in the MI/N-TIMP-3 group, TIMP levels returned to $\mathrm{MI} /$ saline values with the notable exception of endogenous TIMP-3, which increased further post-MI.

Differential Effects of mRNA Levels for Collagen/ Cytokine/Chemokines and Indices of Apoptosis. A robust increase in fibrillar collagen expression occurred in the $\mathrm{MI} /$ saline and MI/N-TIMP-3 groups, which was reduced in the MI/F-TIMP-3 group (Fig. 5). Tumor necrosis factor (TNF) $\alpha$ and CD44 mRNA levels increased in all post-MI groups, but relative TNF $\alpha$ expression was lower in the F-TIMP-3 group. Increased chemokine expression, specifically monocyte chemoattractant protein-1 (MCP1, also known as chemokine ligand 2) and macrophage inflammatory protein-1 alpha (MIP1A, also known as chemokine ligand 3-like 1), increased 

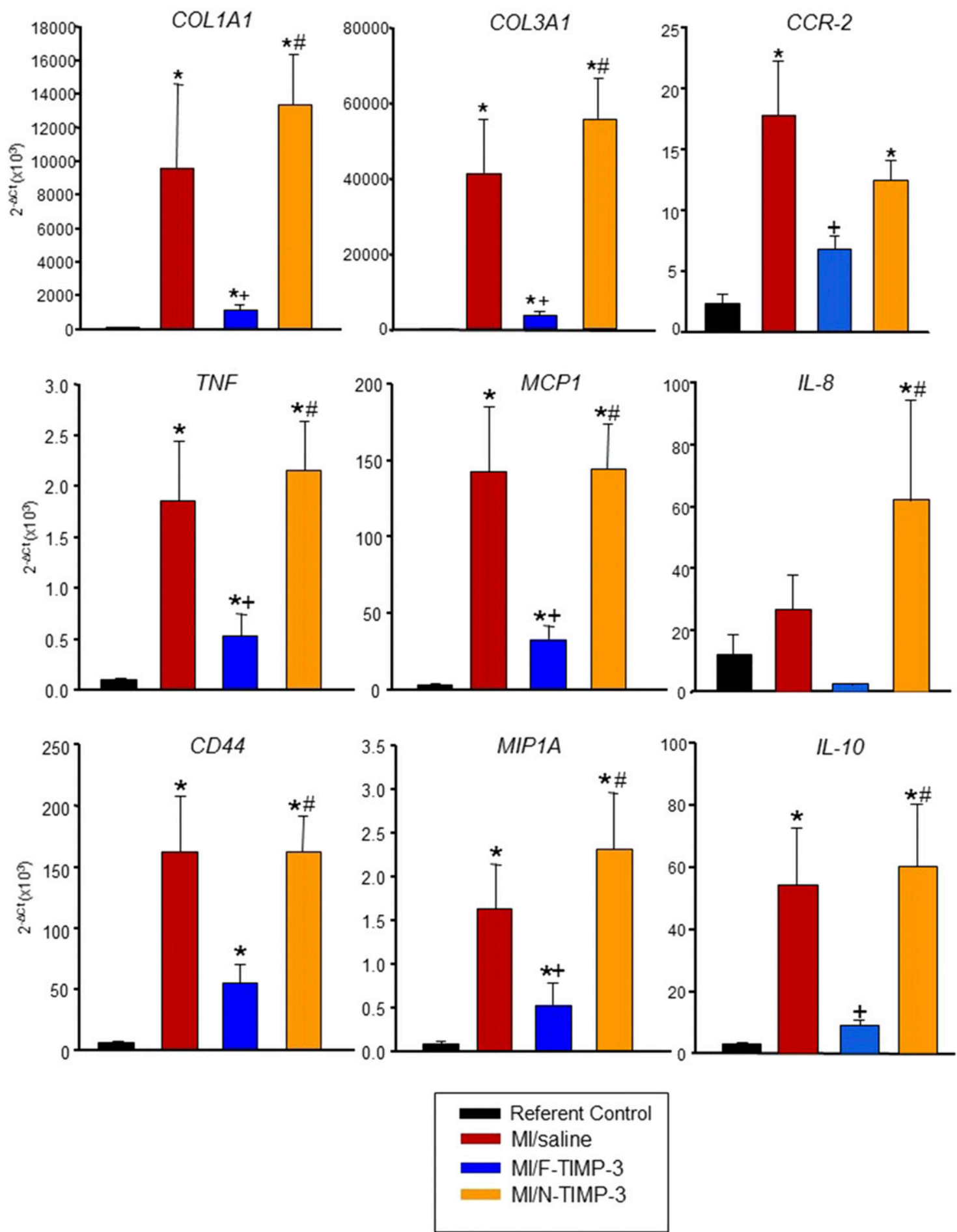

Fig. 5. Relative fibrillar collagen mRNA levels [collagen type I (COL1A1) and collagen type III (COL3A1)] increased post-MI in all groups compared with referent controls, but relative values were lower in the MI/F-TIMP-3 group. Representative mRNA levels from the cytokines and chemokines within the MI regions revealed a robust increase compared with referent controls with similar values between the MI/saline and MI/N-TIMP-3 groups. However, TNF, MCP1, and MIP1A were significantly lower in the MI/F-TIMP-3 group. Using an expanded cytokine array panel (Supplemental Table 2), a number of cytokines were increased post-MI, such as C-C motif receptor-2 (CCR-2) and IL-10, were reduced in the MI/F-TIMP-3 group, and were equivalent or increased from post-MI values in the MI/N-TIMP-3 group. ${ }^{*} P<0.05$ vs. control values; ${ }^{+} P<0.05$ vs. MI/saline; ${ }^{\#} P<0.05$ vs. MI/F-TIMP-3, determined by LSD test. Sample sizes: MI/saline $n=9$, MI/F-TIMP-3 $n=8$, MI/N-TIMP-3 $n=9$, referent control $n=5$.

in all MI groups but were lower in the MI/F-TIMP-3 group (Fig. 5). Relative mRNA levels for proapoptotic BAX and for the antiapoptotic BCL2 were both elevated within the MI region but were significantly reduced in the F-TIMP-3 group (Fig. 6).
Using the full cytokine array, a very similar pattern emerged. Specifically, when the cytokine and cytokine receptor values were pooled and subjected to ANOVA, a significant treatment effect was observed $(\mathrm{F}$ value $=9.6, P<0.001$; Fig. 7A). Moreover, when subjected to pairwise analysis, 

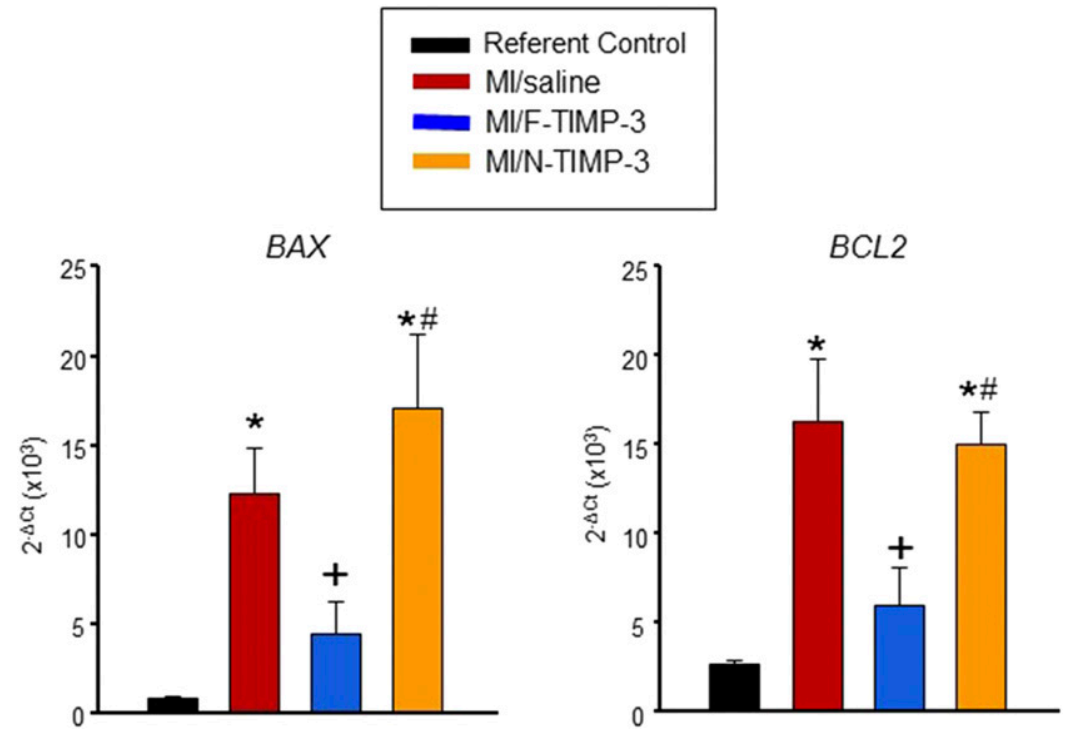

Fig. 6. Targeted PCR measurements of determinants of apoptosis, BAX and BCL2 both increased in the MI/saline and MI/N-TIMP-3 groups and reduced in the MI/F-TIMP-3 group within the MI region. $* P<0.05$ vs. control values; ${ }^{+} P<0.05$ vs. MI/saline; ${ }^{\#} P<0.05$ vs. MI/F-TIMP-3, determined by nonparametric Wilcoxon test. Sample sizes: MI/saline $n=9$, MI/F-TIMP-3 $n=8$, MI/N-TIMP-3 $n=9$, referent control $n=5$. overall cytokine expression increased by over 2 -fold in the MI/ saline group, increased further in the MI/N-TIMP-3 group, and fell to within normal values in the MI/F-TIMP-3 group. Representative selected chemokines, such as C-C motif receptor-2, and interleukins (ILs), such as IL-8 and IL-10, from this overall array are shown in Figure 4, whereby a very similar pattern of expression was observed. Thus, F-TIMP-3 injections, but not N-TIMP-3 injections, reduced the expression of fibrillar collagen and indices of inflammation post-MI.

LV total MMP activity, reflecting a summation of proteolytic activity of all MMP types, was significantly increased within the MI region of all groups with no difference between groups (Fig. 7B). Similarly, MT1-MMP specific activity was increased in all MI groups with a tendency for higher values in both TIMP-3 treatment groups but did not reach statistical significance (Fig. 7C).

Collagen Content and Inflammation After MI; Effects of TIMP-3 Injections. MI sections stained with H\&E revealed inflammatory cells and clear regions of scar formation (Fig. 8). Qualitative assessment of these stained sections revealed a lower overall inflammatory cell infiltrate in the MI/ F-TIMP-3 sections. Clear bands of fibrillar collagen were evident within the MI region with PSR staining (Fig. 8). Quantification of MI and remote sections demonstrated equivalent total fibrillar collagen content within the MI region as well as the remote, nonischemic region for all treatment groups. Thus, although F-TIMP-3 reduced mRNA levels for fibrillar collagen, total collagen content within the MI region remained unaffected, suggesting that changes in posttranscriptional events (i.e., reduced collagen turnover) had occurred. To more carefully examine a potential index of myocardial fibroblast phenotype, SMA histochemistry was performed and identified a robust increase within the MI region for all treatment groups. However, the SMA relative content was reduced in the MI/F-TIMP-3 group. Immunostaining for macrophages (IBA-1) revealed a nominal signal in referent control LV sections, which increased significantly in the MI regions of all treatment groups. The density of staining, likely reflective of macrophage density, was reduced in the MI/F-TIMP-3 group but did not reach statistical significance $(P=0.25)$. Representative histochemical results for referent normal and post-MI sections from the remote region are provided in Supplemental Figure 1.

\section{Discussion}

The post-MI period is invariably accompanied by intense inflammation and ECM remodeling, which is facilitated in part by MMPs (Heymans et al., 1999; Lindsey et al., 2002; Mukherjee et al., 2003; Kandalam et al., 2010). This in turn causes ECM instability and infarct expansion, which is most commonly identified as an increase in LV end-diastolic volume. Although animal models that modify MMP and/or TIMPs have provided a cause-effect relationship to LV remodeling (Heymans et al., 1999; Lindsey et al., 2002; Mukherjee et al., 2003; Spinale, 2007; Kandalam et al., 2010; Lindsey and Zamilpa, 2012), the translation of these basic findings to potential therapeutic strategies has not been forthcoming. The present study addressed this issue and obtained several unique findings. First, injection of a recombinant TIMP-3 into the MI region imparted a beneficial effect in terms of critical determinants of post-MI LV remodeling. Second, post-MI LV remodeling was attenuated with either F-TIMP-3 or N-TIMP-3, suggesting that the N-terminal region of TIMP-3 is sufficient to attenuate early adverse post-MI remodeling. Third, F-TIMP-3 injections, but not N-TIMP-3 injections, reduced relative MMP expression and indices of inflammation at the mRNA level, suggesting that the C-terminal region uniquely affects other biologic pathways.

The progression of post-MI remodeling include LV chamber dilation, thinning of the $\mathrm{LV}$ wall encompassing the $\mathrm{MI}$ region, and a reduction in LV systolic function (Sutton and Sharpe, 2000; Weir et al., 2006; Spinale, 2007; Konstam et al., 2011). The local injection of either a full-length or truncated form of TIMP-3 attenuated all of these indices of post-MI remodeling. Although both TIMP-3 formulations reduced LV dilation, the post-MI trajectory for this index of $L V$ remodeling appeared to be different between the TIMP-3 formulations. In addition, indices of heart failure progression (Colucci and Braunwald, 2005; Zile et al., 2011), such as left atrial size and estimates of pulmonary capillary wedge pressure, were attenuated with F-TIMP-3 or N-TIMP-3 targeted injections. Of note, 

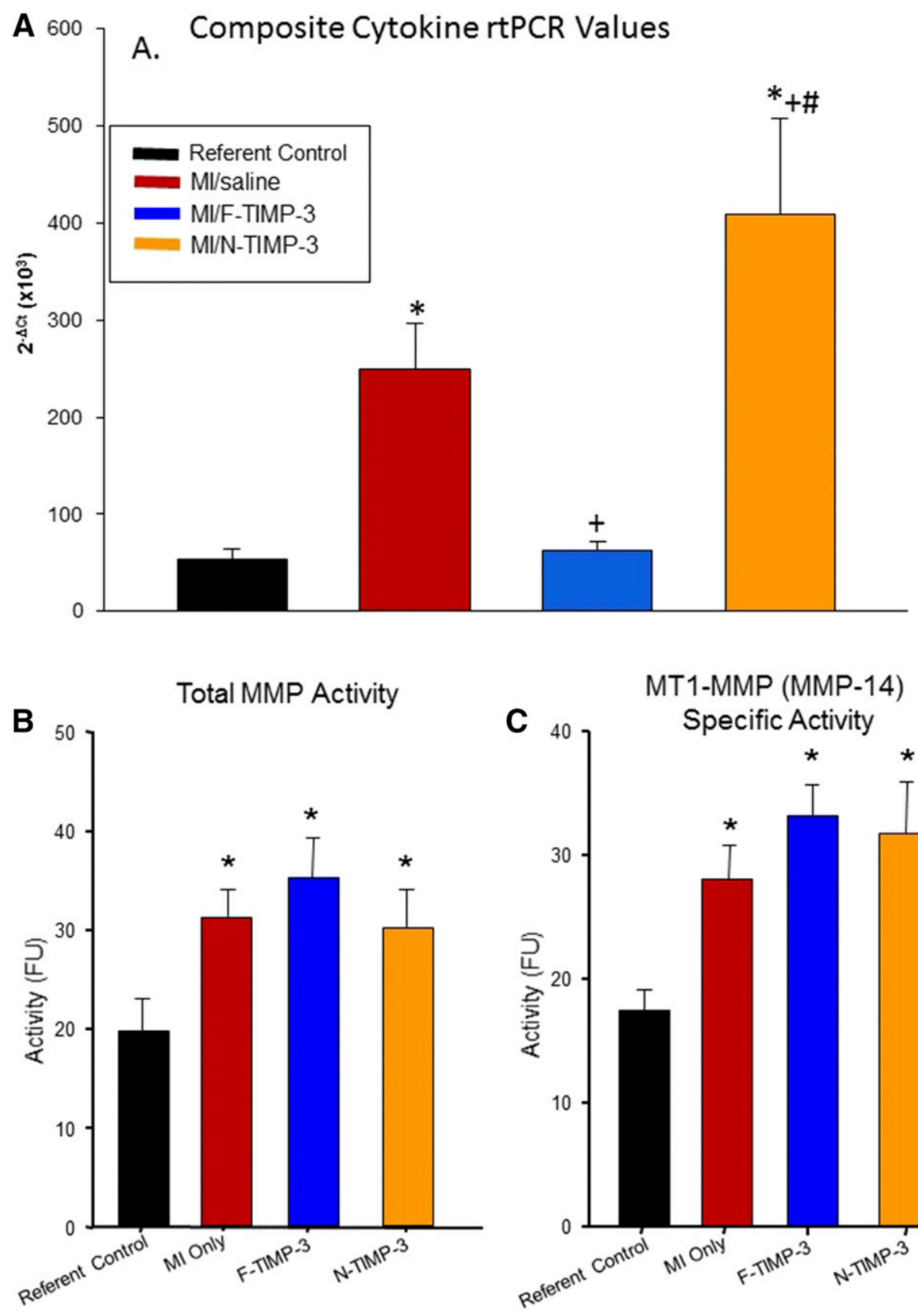

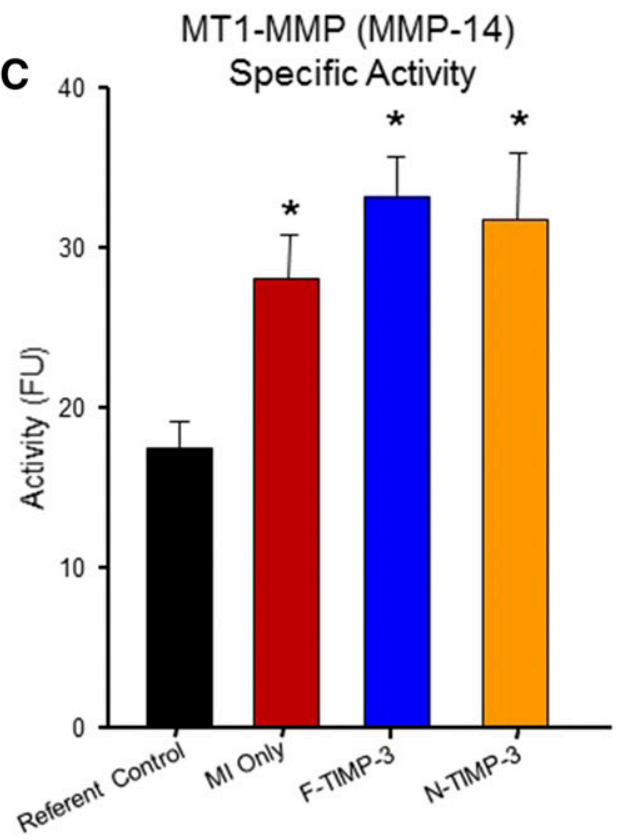

Fig. 7. (A) In addition to targeted PCR cytokine measurements, a full cytokine porcine PCR array was used (Supplemental Table 2), which contained 84 unique cytokines and chemokines. The composite values for all of these measurements are shown here whereby the ANOVA identified a significant overall treatment effect and pairwise comparisons revealed overall cytokine induction in the MI/saline and MI/N-TIMP-3 groups and lower values in the MI/F-TIMP-3 group. The composite cytokine value was the highest in the MI/N-TIMP-3 group. (B) Total MMP activity was determined in LV homogenates taken from the MI region and was increased within the MI region in all groups. (C) MT1-MMP (MMP-14) activity was also determined and revealed a similar pattern to that of total MMP activity. $* P<0.05$ vs. control values; ${ }^{+} P<0.05$ vs. MI/saline; ${ }^{\#} P<0.05$ vs. MI/F-TIMP-3, determined by LSD test. Sample sizes: MI/saline $n=9$, MI/F-TIMP-3 $n=8$, MI/N-TIMP-3 $n=9$, referent $\operatorname{control} n=5$. FU, fluorescence unitsrt; PCR, reverse transcriptionpolymerase chain reaction. pulmonary capillary wedge pressure was higher at early postMI time points with N-TIMP-3 injections, which may have been due to increased myocardial stiffness. However, this remains speculative.

Although both TIMP-3 formulations favorably attenuated post-MI remodeling at the chamber/myocardial level, the mechanism(s) for this effect may have been distinctly different. With respect to MMP/TIMP profiles, F-TIMP-3 reduced MMP and TIMP levels within the MI region, whereas N-TIMP-3 appeared to have either no effect on these expression profiles or arguably caused an amplification of endogenous TIMP-3 levels. However, these changes did not appear to be associated with differences in either global MMP activity or MT1-MMP (MMP-14) specific activity. There are several likely reasons for these observations. Firstly, the retention times for recombinant F-TIMP-3 and N-TIMP-3 were approximately 7 days, and the post-MI samples were collected at 14 days. Thus, there was unlikely to be any retained recombinant TIMP formulation in the MI samples subjected to these MMP activity assays. Secondly, with F-TIMP-3, a relatively concordant reduction in both MMPs and endogenous TIMPs occurred at 14 days post-MI and may have resulted in no net effect on overall proteolytic activity when compared with MI-saline values. It should also be noted that MMP activity in and of itself does not reflect ECM stability or turnover.

In the present study, total fibrillar collagen content as assessed by a quantitative histochemical approach was increased within the $\mathrm{MI}$ and remote regions at 14 days post-MI and was increased to a similar extent with the different TIMP3 formulations. However, it should be recognized that this measurement was taken at one point in time, and whether and 


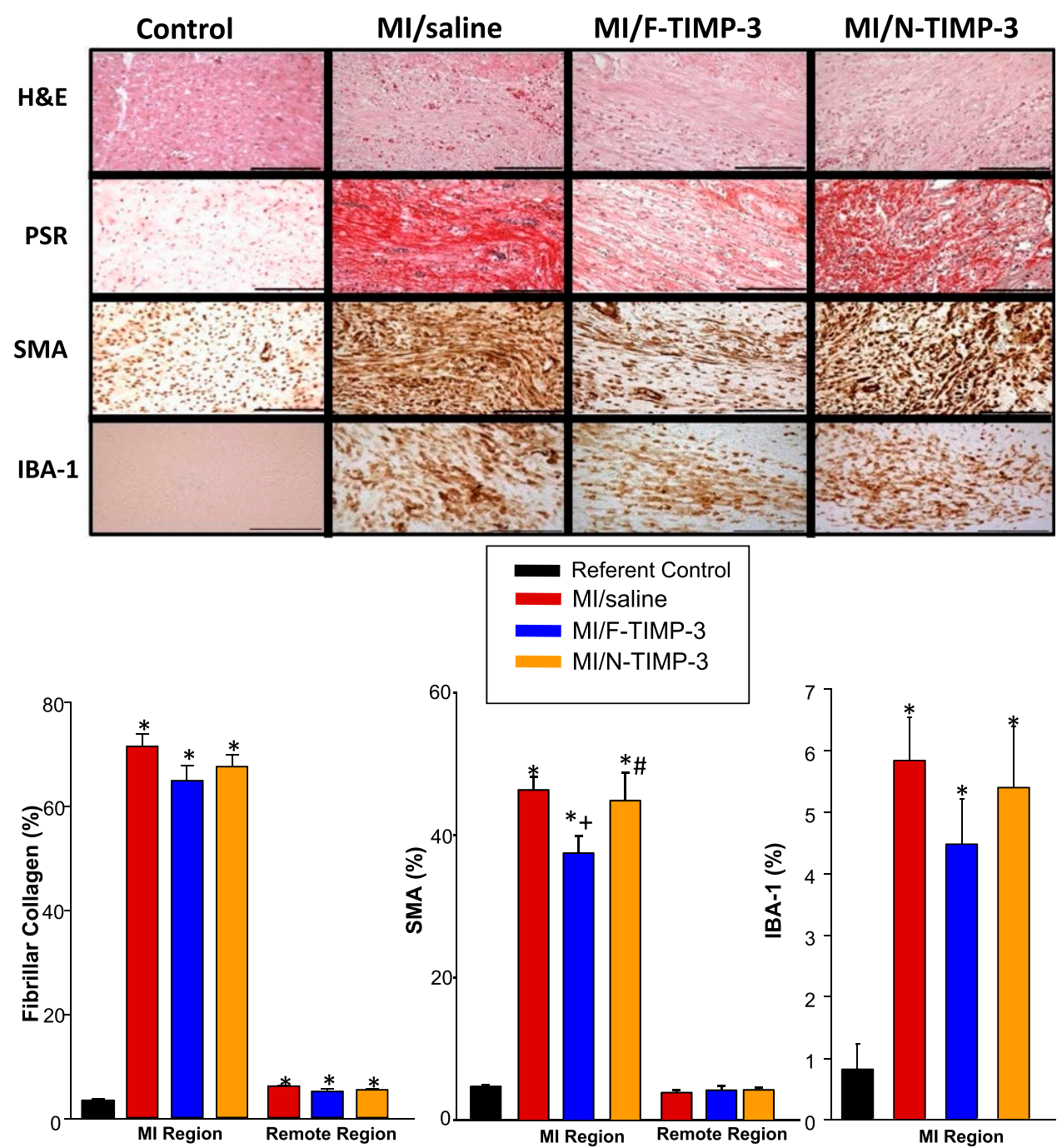

Fig. 8. (Top) H\&E sections revealed a loss of cardiac myocytes within the MI region accompanied by replacement fibrosis and the presence of inflammatory cells. PSR stained sections revealed clear bands of fibrillar collagen within the MI region. SMA staining of the MI region was robustly increased in all groups but appeared reduced in the F-TIMP-3 group. Macrophage staining (IBA antibody) revealed a minimal positive signal in the referent normal samples with a robust increase in all MI sections (original magnification, $40 \times$, Scale bars, $100 \mu \mathrm{m}$ ). (Bottom) Relative collagen content was increased in the MI and remote regions in all treatment groups when compared with referent normal values. Quantitative analysis revealed a robust increase within the MI region for SMA and IBA, which remained elevated in both TIMP-3 groups. However, SMA levels within the MI region were lower in the MI/F-TIMP-3 group. Due to the nominal IBA staining in the remote regions, the quantitative results are not shown. However, representative histologic sections for the remote region are provided in Supplemental Figure 1. $* P<0.05$ vs. control values; ${ }^{+} P<0.05$ vs. $\mathrm{MI} / \mathrm{saline}$; ${ }^{\#} P<0.05$ vs. MI/F-TIMP-3, determined by LSD test. Sample sizes: MI/saline $n=9$, MI/F-TIMP-3 $n=8$, MI/N-TIMP-3 $n=9$, referent control $n=5$.

to what degree fibrillar collagen content was affected, particularly within the $\mathrm{MI}$ region, at earlier time points with either recombinant TIMP-3 formulation remains unknown. The present study did provide evidence that at the transcriptional level, the recombinant TIMP-3 formulations caused differential effects of fibrillar collagen expression within the MI region. Specifically, F-TIMP-3 reduced collagen type I and III mRNA levels at 14 days post-MI, whereas N-TIMP-3 injections did not demonstrate this effect. This underscores the complexity of factors that contribute to fibrillar collagen accumulation within the MI region, and the findings from the present study suggest that certain domains of the TIMP-3 molecule may cause differential effects on the determinants of ECM synthesis and degradation.

A potentially important event is changes in fibroblast phenotype post-MI (Zamilpa et al., 2011; Ma et al., 2013, 2017; Shinde and Frangogiannis, 2014; Khalil et al., 2017; Fu et al., 2018; Wang et al., 2020). TIMP-3 can influence neonatal mouse proliferation, and TIMP-3 deficiency exacerbated postMI cardiac rupture and mortality (Kandalam et al., 2010; Hammoud et al., 2011). It has been established that canonical inflammatory molecules such as TNF can drive fibroblast transdifferentiation (Kassiri et al., 2009; Awad et al., 2010; Ma et al., 2017). Although specific markers of myofibroblasts remain to be fully developed and validated, the identification of SMA expression in these transdifferentiated cells has been used (Awad et al., 2010; Ma et al., 2017). The findings of the present study would suggest that the $\mathrm{N}$-terminal region of N-TIMP-3 was insufficient to attenuate this marker of fibroblast activation post-MI. However, the present study used in situ markers for fibroblast activation and future studies utilizing primary myofibroblast cultures, as described in previous studies (Hammoud et al., 2011; Wang et al., 2020), would add scientific insight to this issue.

It has been shown previously that prolonged inflammation, in particular abnormalities in macrophage activation and polarization, can contribute to adverse post-MI remodeling (Zamilpa et al., 2011; Ma et al., 2013). The predominant observation from the present study was that F-TIMP-3 injections, but not N-TIMP-3 injections, significantly reduced indices of inflammation. A determinant of macrophage density (CD44) (Frangogiannis, 2012) was selectively reduced by F-TIMP-3 injections. Moreover, markers of macrophage maturation and infiltration (Dewald et al., 2005), MCP1 and MIP1A, increased within the MI region and were markedly lower with F-TIMP-3 injections. It has been reported previously in vitro that distinct functional domains exist within the TIMP-3 molecule that affect ECM proteolytic pathways 
and that of cytokine processing/induction (Wei et al., 2005; Troeberg et al., 2009; Bourboulia and Stetler-Stevenson, 2010; Brew and Nagase, 2010). For example, Wei et al. (2005) reported that mutations in the N-terminal region of TIMP-3 significantly impaired MMP inhibition, but not effects upon cytokine processing (adamalysin (ADAM) dependent), implying that a domain outside of the $\mathrm{N}$-terminal region is responsible for this biologic activity. Past studies of TIMP-3 gene deletion in mice demonstrated not only an acceleration of adverse myocardial remodeling, but also an association with increased cytokine expression, notably TNF, providing further evidence for the duality of function of TIMP-3 (Kandalam et al., 2010; Hammoud et al., 2011). Inhibition of the initial inflammatory/wound healing response after MI can cause detrimental effects on post-MI remodeling (Spinale, 2007; Lindsey and Zamilpa, 2012; Ma et al., 2013). Thus, the attenuation of specific determinants of macrophage activation/polarization, such as MCP1 and MIP1A, which was achieved by F-TIMP-3 but not N-TIMP-3, may not necessarily be beneficial. The present study using high sensitivity troponin measurements identified an equivalent degree of initial myocardial injury in all groups, and indices of apoptosis shifted in all three groups at 14 days post-MI. F-TIMP-3 injections directionally reduced both Bax (proapoptotic) and BCL2 (antiapoptotic) at 14 days post-MI and thus may have not altered the stoichiometric balance of this specific apoptotic cascade. Whether and to what degree TIMP-3 injections may influence myocardial viability pathways post-MI remains unclear. Furthermore, although the present study identified injections of the full-length TIMP-3 attenuated indices of inflammation post-MI at the transcriptional level, the downstream effects on inflammation remains to be fully explored.

The effects on post-MI remodeling achieved in the present study using local injection of TIMP-3 formulations appeared equivalent to that reported with systemic pharmacological MMP inhibition (Webb et al., 2006; Weir et al., 2006; Awad et al., 2010; Konstam et al., 2011; Zamilpa et al., 2011; Bolz et al., 2016; Ma et al., 2017). Although the regulation of MMP activity has been shown to be a relevant therapeutic target in a number of tissue remodeling processes, such as cancer, rheumatoid, and cardiovascular disease, systemic delivery of pharmacological MMP inhibitors remains a significant challenge (Peterson, 2004; Dorman et al., 2010). In terms of post-MI remodeling, a pharmacological MMP inhibitor was advanced to initial clinical trials (Hudson et al., 2006). In an initial study utilizing a pharmacological MMP inhibitor in post-MI patients, a suboptimal dosing schedule was used due to concerns regarding systemic effects, and thus the likelihood of achieving local MMP inhibition was minimal (Hudson et al., 2006; Spinale, 2007). There are inherent limitations of the present study that must be acknowledged. First, this study used a single time in the myocardial tissue that was quite short (days), and our initial injection studies demonstrated that only a small amount of the injected TIMP-3 formulation remained over a time course relevant to the present study period. The relative retention time of the N-TIMP-3 was shorter than the F-TIMP-3 and was likely due to the lower molecular weight of this truncated TIMP-3 molecule as well as the fact that ECM binding domains contained within the F-TIMP-3 molecule were absent. This laboratory reported the use of a slow release hydrogel construct (Eckhouse et al., 2014) that may improve localized release of TIMP-3 formulations.
Finally, the present study used a coronary ligation approach to induce a reproducible and well defined MI region (Mukherjee et al., 2008; Eckhouse et al., 2014). However, a more common clinical event is an ischemic episode followed by reperfusion in which the extent and type of inflammation and MMP activation may be quite different. Nevertheless, these proof-ofconcept studies clearly demonstrate the feasibility and early efficacy of this therapeutic approach in terms of interrupting the course of adverse LV remodeling post-MI.

\section{Authorship Contributions}

Participated in research design: Lobb, Brower, Khakoo, Lee, Spinale.

Conducted experiments: Lobb, Doviak, Brower, Romito, Shuman, Freels, Zellars, Freeburg, Spinale.

Contributed new reagents or analytic tools: O'Neill, Smith, Khakoo, Lee.

Performed data analysis: Doviak, Romito, Shuman, Freels, Zellars, Freeburg, Spinale.

Wrote or contributed to the writing of the manuscript: Lobb, Lee, Spinale.

\section{References}

Abbate A, Biondi-Zoccai GGL, and Baldi A (2002) Pathophysiologic role of myocardial apoptosis in post-infarction left ventricular remodeling. J Cell Physiol 193: $145-153$.

Awad AE, Kandalam V, Chakrabarti S, Wang X, Penninger JM, Davidge ST, Oudit GY, and Kassiri Z (2010) Tumor necrosis factor induces matrix metalloproteinases in cardiomyocytes and cardiofibroblasts differentially via superoxide production in a PI3Kgamma-dependent manner. Am J Physiol Cell Physiol 298:C679-C692.

Bolz M, Ruggli N, Borel N, Pluschke G, and Ruf MT (2016) Local cellular immune responses and pathogenesis of buruli ulcer lesions in the experimental Mycobacterium ulcerans pig infection model. PLoS Negl Trop Dis 10:e0004678.

Bourboulia D and Stetler-Stevenson WG (2010) Matrix metalloproteinases (MMPs) and tissue inhibitors of metalloproteinases (TIMPs): positive and negative regulators in tumor cell adhesion. Semin Cancer Biol 20:161-168.

Brew K and Nagase H (2010) The tissue inhibitors of metalloproteinases (TIMPs): an ancient family with structural and functional diversity. Biochim Biophys Acta 1803:55-71.

Colucci WS and Braunwald E (2005) Pathophysiology of heart failure. Braunwald's Heart Disease: A Textbook of Cardiovascular Medicine 509-538, Elsevier, Philadelphia.

Dewald O, Zymek P, Winkelmann K, Koerting A, Ren G, Abou-Khamis T, Michae LH, Rollins BJ, Entman ML, and Frangogiannis NG (2005) CCL2/Monocyte chemoattractant protein-1 regulates inflammatory responses critical to healing myocardial infarcts. Circ Res 96:881-889.

Dixon JA and Spinale FG (2009) Large animal models of heart failure: a critical link in the translation of basic science to clinical practice. Circ Heart Fail 2:262-271.

Dormán G, Cseh S, Hajdú I, Barna L, Kónya D, Kupai K, Kovács L, and Ferdinandy P (2010) Matrix metalloproteinase inhibitors: a critical appraisal of design principles and proposed therapeutic utility. Drugs 70:949-964.

Douglas DA, Shi YE, and Sang QA (1997) Computational sequence analysis of the tissue inhibitor of metalloproteinase family. J Protein Chem 16:237-255.

Eckhouse SR, Purcell BP, McGarvey JR, Lobb D, Logdon CB, Doviak H, O'Neill JW, Shuman JA, Novack CP, Zellars KN, et al. (2014) Local hydrogel release of recombinant TIMP-3 attenuates adverse left ventricular remodeling after experimental myocardial infarction. Sci Transl Med 6:223ra21.

Frangogiannis NG (2012) Regulation of the inflammatory response in cardiac repair. Circ Res 110:159-173.

Fu X, Khalil H, Kanisicak O, Boyer JG, Vagnozzi RJ, Maliken BD, Sargent MA Prasad V, Valiente-Alandi I, Blaxall BC, et al. (2018) Specialized fibroblast differentiated states underlie scar formation in the infarcted mouse heart. $J$ Clin Invest 128:2127-2143.

Hammoud L, Burger DE, Lu X, and Feng Q (2009) Tissue inhibitor of metalloproteinase-3 inhibits neonatal mouse cardiomyocyte proliferation via EGFR/JNK/SP-1 signaling. Am J Physiol Cell Physiol 296:C735-C745.

Hammoud L, Lu X, Lei M, and Feng Q (2011) Deficiency in TIMP-3 increases cardiac rupture and mortality post-myocardial infarction via EGFR signaling: beneficial effects of cetuximab. Basic Res Cardiol 106:459-471.

Heymans S, Luttun A, Nuyens D, Theilmeier G, Creemers E, Moons L, Dyspersin GD, Cleutjens JP, Shipley M, Angellilo A, et al. (1999) Inhibition of plasminogen activators or matrix metalloproteinases prevents cardiac rupture but impairs therapeutic angiogenesis and causes cardiac failure. Nat Med 5:1135-1142.

Hudson MP, Armstrong PW, Ruzyllo W, Brum J, Cusmano L, Krzeski P, Lyon R, Quinones M, Theroux P, Sydlowski D, et al. (2006) Effects of selective matrix metalloproteinase inhibitor (PG-116800) to prevent ventricular remodeling after myocardial infarction: results of the PREMIER (Prevention of Myocardial Infarction Early Remodeling) trial. J Am Coll Cardiol 48:15-20.

Kandalam V, Basu R, Abraham T, Wang X, Awad A, Wang W, Lopaschuk GD, Maeda N, Oudit GY, and Kassiri Z (2010) Early activation of matrix metalloproteinases underlies the exacerbated systolic and diastolic dysfunction in mice lacking TIMP3 
following myocardial infarction. Am $J$ Physiol Heart Circ Physiol 299: H1012-H1023.

Kassiri Z, Defamie V, Hariri M, Oudit GY, Anthwal S, Dawood F, Liu P, and Khokha R (2009) Simultaneous transforming growth factor beta-tumor necrosis factor activation and cross-talk cause aberrant remodeling response and myocardial fibrosis in Timp3-deficient heart. J Biol Chem 284:29893-29904.

Khalil H, Kanisicak O, Prasad V, Correll RN, Fu X, Schips T, Vagnozzi RJ, Liu R, Huynh T, Lee SJ, et al. (2017) Fibroblast-specific TGF- $\beta$-Smad2/3 signaling underlies cardiac fibrosis. J Clin Invest 127:3770-3783.

Konstam MA, Kramer DG, Patel AR, Maron MS, and Udelson JE (2011) Left ventricular remodeling in heart failure: current concepts in clinical significance and assessment. JACC Cardiovasc Imaging 4:98-108.

Leco KJ, Khokha R, Pavloff N, Hawkes SP, and Edwards DR (1994) Tissue inhibitor of metalloproteinases-3 (TIMP-3) is an extracellular matrix-associated protein with a distinctive pattern of expression in mouse cells and tissues. J Biol Chem 269:9352-9360.

Lindsey ML, Gannon J, Aikawa M, Schoen FJ, Rabkin E, Lopresti-Morrow L, Crawford J, Black S, Libby P, Mitchell PG, et al. (2002) Selective matrix metalloproteinase inhibition reduces left ventricular remodeling but does not inhibit angiogenesis after myocardial infarction. Circulation 105:753-758.

Lindsey ML and Zamilpa R (2012) Temporal and spatial expression of matrix metalloproteinases and tissue inhibitors of metalloproteinases following myocardial infarction. Cardiovasc Ther 30:31-41.

Lovelock JD, Baker AH, Gao F, Dong JF, Bergeron AL, McPheat W, Sivasubramanian N, and Mann DL (2005) Heterogeneous effects of tissue inhibitors of matrix metalloproteinases on cardiac fibroblasts. Am J Physiol Heart Circ Physiol 288:H461-H468.

Lu Y, Liu S, Zhang S, Cai G, Jiang H, Su H, Li X, Hong Q, Zhang X, and Chen X (2011) Tissue inhibitor of metalloproteinase-1 promotes NIH3T3 fibroblast proliferation by activating p-Akt and cell cycle progression. Mol Cells 31:225-230.

Ma Y, Halade GV, Zhang J, Ramirez TA, Levin D, Voorhees A, Jin YF, Han HC Manicone AM, and Lindsey ML (2013) Matrix metalloproteinase-28 deletion exacerbates cardiac dysfunction and rupture after myocardial infarction in mice by inhibiting M2 macrophage activation. Circ Res 112:675-688.

Ma Y, Iyer RP, Jung M, Czubryt MP, and Lindsey ML (2017) Cardiac fibroblast activation post-myocardial infarction: current knowledge gaps. Trends Pharmacol Sci 38:448-458.

Melendez-Zajgla J, Del Pozo L, Ceballos G, and Maldonado V (2008) Tissue inhibitor of metalloproteinases-4. The road less traveled. Mol Cancer 7:85.

Moore L, Fan D, Basu R, Kandalam V, and Kassiri Z (2012) Tissue inhibitor of metalloproteinases (TIMPs) in heart failure. Heart Fail Rev 17:693-706.

Mukherjee R, Brinsa TA, Dowdy KB, Scott AA, Baskin JM, Deschamps AM, Lowry AS, Escobar GP, Lucas DG, Yarbrough WM, et al. (2003) Myocardial infarct expansion and matrix metalloproteinase inhibition. Circulation 107:618-625.

Mukherjee R, Zavadzkas JA, Saunders SM, McLean JE, Jeffords LB, Beck C, Stroud RE, Leone AM, Koval CN, Rivers WT, et al. (2008) Targeted myocardial microinjections of a biocomposite material reduces infarct expansion in pigs. Ann Thorac Surg 86:1268-1276.

Peterson JT (2004) Matrix metalloproteinase inhibitor development and the remodeling of drug discovery. Heart Fail Rev 9:63-79.

Peterson JT, Li H, Dillon L, and Bryant JW (2000) Evolution of matrix metalloprotease and tissue inhibitor expression during heart failure progression in the infarcted rat. Cardiovasc Res 46:307-315.
Shinde AV and Frangogiannis NG (2014) Fibroblasts in myocardial infarction: a role in inflammation and repair. $J \mathrm{Mol}$ Cell Cardiol 70:74-82.

Spinale FG (2007) Myocardial matrix remodeling and the matrix metalloproteinases: influence on cardiac form and function. Physiol Rev 87:1285-1342.

Spinale FG, Koval CN, Deschamps AM, Stroud RE, and Ikonomidis JS (2008) Dynamic changes in matrix metalloprotienase activity within the human myocardial interstitium during myocardial arrest and reperfusion. Circulation 118 (14 Suppl): S16-S23.

Sutton MG and Sharpe N (2000) Left ventricular remodeling after myocardial infarction: pathophysiology and therapy. Circulation 101:2981-2988.

Troeberg L, Fushimi K, Scilabra SD, Nakamura H, Dive V, Thøgersen IB, Enghild JJ, and Nagase H (2009) The C-terminal domains of ADAMTS-4 and ADAMTS-5 promote association with N-TIMP-3. Matrix Biol 28:463-469.

Wang X, Morelli MB, Matarese A, Sardu C, and Santulli G (2020) Cardiomyocytederived exosomal microRNA-92a mediates post-ischemic myofibroblast activation both in vitro and ex vivo. ESC Heart Fail 7:284-288.

Webb CS, Bonnema DD, Ahmed SH, Leonardi AH, McClure CD, Clark LL, Stroud RE, Corn WC, Finklea L, Zile MR, et al. (2006) Specific temporal profile of matrix metalloproteinase release occurs in patients after myocardial infarction: relation to left ventricular remodeling. Circulation 114:1020-1027.

Wei S, Kashiwagi M, Kota S, Xie Z, Nagase H, and Brew K (2005) Reactive site mutations in tissue inhibitor of metalloproteinase-3 disrupt inhibition of matrix metalloproteinases but not tumor necrosis factor-alpha-converting enzyme. J Biol Chem 280:32877-32882.

Weir RA, McMurray JJ, and Velazquez EJ (2006) Epidemiology of heart failure and left ventricular systolic dysfunction after acute myocardial infarction: prevalence, clinical characteristics, and prognostic importance. Am J Cardiol 97:13F-25F

Wilson EM, Moainie SL, Baskin JM, Lowry AS, Deschamps AM, Mukherjee R, Guy TS, St John-Sutton MG, Gorman JH III, Edmunds LH Jr., et al. (2003) Region- and type-specific induction of matrix metalloproteinases in post-myocardial infarction remodeling. Circulation 107:2857-2863.

Yang TT and Hawkes SP (1992) Role of the 21-kDa protein TIMP-3 in oncogenic transformation of cultured chicken embryo fibroblasts. Proc Natl Acad Sci USA 89 10676-10680.

Yu WH, Yu S, Meng Q, Brew K, and Woessner JF Jr. (2000) TIMP-3 binds to sulfated glycosaminoglycans of the extracellular matrix. J Biol Chem 275:31226-31232.

Zamilpa R, Kanakia R, Cigarroa J IV, Dai Q, Escobar GP, Martinez H, Jimenez F, Ahuja SS, and Lindsey ML (2011) CC chemokine receptor 5 deletion impairs macrophage activation and induces adverse remodeling following myocardial infarction. Am J Physiol Heart Circ Physiol 300:H1418-H1426.

Zile MR, Desantis SM, Baicu CF, Stroud RE, Thompson SB, McClure CD, Mehurg SM, and Spinale FG (2011) Plasma biomarkers that reflect determinants of matrix composition identify the presence of left ventricular hypertrophy and diastolic heart failure. Circ Heart Fail 4:246-256.

Address correspondence to: Dr. Francis G. Spinale, Cardiovascular Translational Research Center, University of South Carolina School of Medicine, 6439 Garners Ferry Road, Building 3, Room 234, Columbia, SC 29208. E-mail: cvctrc@uscmed.sc.edu 